\title{
A minimal model of tumor growth with angiogenic inhibition using bevacizumab
}

\author{
Dániel András Drexler*, Johanna Sápi*, Levente Kovács* \\ ${ }^{*}$ Research and Innovation Center of Óbuda University \\ Physiological Controls Research Center, Óbuda University, Hungary \\ (e-mails: \{drexler.daniel,sapi.johanna,kovacs.levente\}@nik.uni-obuda.hu).
}

\begin{abstract}
Modeling the tumor growth under angiogenic inhibition is an important step towards designing tumor treatment therapies based on mathematical tools. Our goal is to create a model for tumor growth that describes the underlying physiological processes adequately while being as simple as possible. We propose a second-order model containing linear terms and one bilinear term modeling the dynamics of tumor volume and inhibitor level, and work out the parametric identification process for the model. The parametric identification of the model is done using measurements from experiments on $\mathrm{C} 57 \mathrm{Bl} / 6$ mice with $\mathrm{C38}$ colon adenocarcinoma treated with bevacizumab. The control group of the mice received one injection at the beginning of the experiment, these measurement data are used for parametric identification, while the case group of mice received injection at each day of the treatment, these measurements are used to validate the model. The validation showed that the proposed model is capable of describing the tumor growth dynamics.

Index Terms-Tumor growth model, Angiogenic inhibition, Bevacizumab, Minimal model
\end{abstract}

\section{INTRODUCTION}

Angiogenesis (formation of new blood vessels) is a physiological process that helps tumors grow over a certain size and can be inhibited with the application of the appropriate drugs [1]. Inhibiting angiogenesis thus indirectly affects tumor growth and is a potential therapeutic tool that can be applied against cancer mechanisms [2]. However, in clinical practice the optimal therapeutic protocol is still unknown [3]. Creating a model-based optimal protocol could significantly help to increase the quality of cancer treatments, and has been investigated by many authors [4], [5], [6], [7]. However, all these control design methods require a practical model describing the physiology of tumor growth and the effect of the inhibitor on the tumor growth.

There are different tumor growth models that can be found in the literature, from the simplest models using linear dynamics to complex models with nonlinear terms in their differential equations [8]. The advantage of the linear models is their simplicity, their disadvantage is that they usually can not describe physiologically important phenomena. The advantage of nonlinear complex models is that they can describe many physiologically important phenomena, however they do that at the expense of the usability of the models.

The most widely used and accepted model that defines tumor growth under angiogenic inhibition was proposed by Hahnfeldt at al [9]. This is a second-order model, thus relatively simple, but uses nonlinear terms in the differential equations. Tylcz at al. gave a model of the dynamics of the vascularization of the tumor under treatment with angiogenic inhibitor in [10], mainly describing the dynamics of the mode of action of the inhibitor.

In [11] it was shown that linear dynamics can describe the measurements for tumor growth without any therapy. However, it turned out in [12] that the linear dynamics may fail if there is therapy, since the effect of therapy and the self-induced growth of the tumor can not be distinguished if a linear model is used to describe the dynamics. We propose a simple secondorder model in Section II were the only nonlinear term in the differential equations is the one describing the effect of the inhibition in order to solve this problem.

The model proposed here uses linear pharmakokinetics to describe the level of the inhibitor in the patient, linear dynamics to define the growth of the tumor, and bilinear dynamics to define the inhibition of tumor growth by the drug. The symbolic solution to the differential equations governing the tumor growth dynamics can be written explicitly if there is only one injection at the beginning of the treatment. This explicit result can be linearized in the parameters and linear parameter estimation can be carried out with least squares estimation as explained in Section III.

The parametric identification of the model is done using measurements from $\mathrm{C} 57 \mathrm{Bl} / 6$ mice experiments, where the mice were implanted subcutaneously with C38 colon adenocarcinoma, and received treatment with bevacizumab [13]. The mice were separated into a control group and a case group. The mice in the control group received only one injection at the beginning of the treatment, while the mice in the case group received injection at each day of the treatment. The parametric identification of the proposed model is done using the measurements from the control group, and the results are validated using the measurements from the case group in Section IV.

The model given in Section II is a simple model that is capable to express the effect of inhibitor separately from the growth mechanism of the tumor, as it will be shown in Section IV. Moreover, the validation of the model shows that it describes the measurements appropriately. 


\section{A MINIMAL MODEL OF TUMOR GROWTH UNDER ANGIOGENIC INHIBITION}

The dynamics of the proposed model of tumor growth is governed by the differential equations

$$
\begin{aligned}
\dot{x} & =a x-b x y \\
\dot{y} & =-c y
\end{aligned}
$$

where $x$ is the time function of tumor volume given in $\mathrm{mm}^{3}$, $y$ is the time function of the level of inhibitor in the host given in $\mathrm{mg} / \mathrm{kg}$ (i.e. $\mathrm{mg}$ of inhibitor per body mass $\mathrm{kg}$ of the host), and the parameters of the model are

- $a$ : the tumor growth rate, measured in $1 /$ day;

- $b$ : the inhibition rate, measured in $\mathrm{kg} /(\mathrm{mg} \cdot$ day);

- $c$ : the clearance of the inhibitor, measured in $1 /$ day.

Equation (2) defines the linear pharmakokinetics of the inhibitor. According to this equation, the depletion of the inhibitor is governed by a linear differential equation, thus its time function is given by an exponential function

$$
y(t)=y(0) e^{-c t}
$$

with $y(0)$ being the initial condition. Therefore, if the amount of injection $y(0)$ is given to the patient, the level of the inhibitor in the patient is described by (3) if there was no inhibitor present in the patient before the injection. The parameter $c$ is the clearance of the inhibitor. If we know the half-life of the inhibitor denoted by $T_{1 / 2}$, then the clearance can be calculated as

$$
c=\frac{\ln 2}{T_{1 / 2}} .
$$

Equation (1) describes the dynamics of tumor growth. The first term on the right-hand side of (1) specifies exponential growth of tumor volume with growth parameter $a$. This term defines an unstable system if $a$ is positive, i.e. the tumor grows uncontrollably (described by an exponential function), and there is no upper bound for the tumor volume. Note that the lack of upper bound is considered as a disadvantage of exponential tumor growth models, however we found that this model fits the measurements adequately. The second term on the right-hand side of (1) defines the inhibition effect of the applied drug onto the tumor growth. This term depends on the product of the tumor volume and the inhibitor level, indicating that if there is no tumor, then there is no inhibiting effect regardless of the amount of inhibitor present in the host. This bilinear term is the most simple term that can describe this phenomenon. The rate of inhibition is the constant $b$, and since the sign of the second term is negative, $b$ is positive if the inhibitor slows down the growth process (thus inhibits tumor growth).

The solution of the differential equation (1) is

$$
x(t)=x(0) e^{a t-b y_{0} / c\left(e^{-c t}-1\right)},
$$

with $x(0)$ being the initial tumor volume and $y(0)$ being the amount of drug injected at time 0 , provided that there were no inhibitor present in the host before injection.
The model given by (1)-(2) is equivalent to the fictive chemical reaction given by the following reaction steps with species $\mathrm{X}$ representing the tumor volume and the species $\mathrm{Y}$ representing the inhibitor level:

- $\mathrm{O} \stackrel{\mathrm{a}}{\longrightarrow} \mathrm{X}$ that defines that there is an inner flow of the species $\mathrm{X}$ with a reaction rate coefficient $a$ from an outer species $\mathrm{O}$ (that represents the species outside the region of the model);

- $\mathrm{Y} \stackrel{\mathrm{c}}{\longrightarrow} \mathrm{O}$ that defines that there is an outflow of the species $\mathrm{Y}$ with a reaction rate coefficient $c$;

- $\mathrm{X}+\mathrm{Y} \stackrel{\mathrm{b}}{\longrightarrow} \mathrm{Y}$ that defines that the species $\mathrm{X}$ and $\mathrm{Y}$ react and after the reaction the species $X$ disappears with a reaction rate coefficient $b$.

The connection of this chemical reaction and the differential equations (1)-(2) can be described by the methods that can be found e.g. in [14], [15].

We emphasize here that the model described by (1)-(2) is the simplest model that specifies the tumor growth dynamics under angiogenic inhibition, capturing the following phenomena:

- it describes (unstable) tumor growth;

- it describes inhibition of tumor growth by the drug, while retaining the positivity of the model;

- it describes the linear pharmakokinetics of the inhibitor.

However, the proposed model can not capture the following phenomena that are physiologically important:

- giving an upper bound for the tumor growth;

- describing the indirect effect of the inhibitor on tumor growth through modeling the dynamics of the supporting vasculature.

Most tumor models incorporate the description of these phenomena into the model too, however we have found that the proposed model explains the measurements well without these phenomena being incorporated into the model, as it will be shown in Section IV.

The only equilibrium point of the model given by (1)-(2) is the trivial equilibrium, i.e. the equations

$$
\begin{aligned}
& 0=a x_{\infty}-b x_{\infty} y_{\infty} \\
& 0=-c y_{\infty}
\end{aligned}
$$

are satisfied only at $x_{\infty}=0$ and $y_{\infty}=0$. This equilibrium is unstable, since the Jacobian of the system of differential equations (1)-(2) at the equilibrium point is

$$
\begin{aligned}
\left.\left(\begin{array}{c}
a x-b x y \\
-c y
\end{array}\right)\right|_{x=0, y=0} & =\left.\left(\begin{array}{cc}
a-b y & -b x \\
0 & -c
\end{array}\right)\right|_{x=0, y=0} \\
& =\left(\begin{array}{cc}
a & 0 \\
0 & -c
\end{array}\right)
\end{aligned}
$$

that has a positive eigenvalue if $a>0$, which implies unstable tumor growth without inhibition. This can be interpreted such that if we give only one injection at the beginning of the treatment, the tumor volume will not be stabilized in an equilibrium, but it will grow with growth rate $a$ after the inhibitor is depleted. 
However, if we extend the inhibitor dynamics by adding an inhibitor inflow rate $I$ (e.g. to model further injections or infusion), i.e. (2) becomes

$$
\dot{y}=-c y+I,
$$

then the equilibria of the model are the solutions to

$$
\begin{aligned}
& 0=a x_{\infty}-b x_{\infty} y_{\infty} \\
& 0=-c y_{\infty}+I_{\infty}
\end{aligned}
$$

that are at

$$
\begin{aligned}
y_{\infty} & =\frac{a}{b} \\
I_{\infty} & =c \frac{a}{b}
\end{aligned}
$$

with $x_{\infty} \in \mathbb{R}^{+}$. Thus if there is an exogenous inhibitor dosage, then the equilibrium is independent of the tumor volume, and only depends on the parameters of the model. Note that we will use the model without the input $I$ in the parametric identification in Section III, since we will only have one injection at the beginning of the measurements that can be modeled as an initial condition for (2).

\section{PARAMETRIC IDENTIFICATION OF THE MODEL USING LEAST SQUARES ESTIMATION}

The measurements used for parametric identification of the model are from experiments where $\mathrm{C} 57 \mathrm{Bl} / 6$ mice were implanted subcutaneously by C38 colon adenocarcinoma, and the tumor volumes were measured every two days starting from three days after the implantation. In the control group, mice received one injection of $10 \mathrm{mg} / \mathrm{kg}$ bevacizumab at the third day. In the case group, mice received $1 / 18 \mathrm{mg} / \mathrm{kg}$ injection each day starting from the third day for 18 days. The tumor volumes were measured with calipers and MRI, the calculation of tumor volume based on these measurements and more details of the experiment can be found in [13], [16]. The parametric identification was done by utilizing the measurement from the control group, while validation of the model was done using the measurements from the case group.

Since the injection was at the third day of the experiment, and the measurements were also carried out starting from the third day, we will treat the third day as $t=0$ day in the mathematical description. The parametric identification is carried out using the measurements from the control group where the mice received only one injection, and there were no inhibitor present in the mice before the injection, thus the tumor volume can be described by

$$
x(t)=x(0) e^{a t-10 b / c\left(e^{-c t}-1\right)},
$$

which can be acquired by substituting $y(0)=10 \mathrm{mg} / \mathrm{kg}$ into (5). We know from [17] that the half-life of the inhibitor bevacizumab is 3.9 days if it is injected intravenously into mice, thus the clearance of the inhibitor is $c=\ln (2) / 3.91 /$ day. We suppose that this clearance is valid in our measurements as well. Thus, the remaining unknown parameters in (14) are the parameters $x(0), a$, and $b$. These parameters can be acquired using least squares estimation.

Equation (14) can be linearized in the parameters by taking the logarithm of both sides resulting in

$$
\ln x(t)=\ln x(0)+a t-10 b / c\left(e^{-c t}-1\right)
$$

that can be rearranged to get

$$
\ln x(t)=\left(\begin{array}{ccc}
1 & t & -10 / c\left(e^{-c t}-1\right)
\end{array}\right)\left(\begin{array}{c}
\ln x(0) \\
a \\
b
\end{array}\right)
$$

For each mouse, there are ten measurements carried out in times $T_{0}, T_{1}, \ldots, T_{9}$ with $T_{i}=2 i$ days, provided that the third day of the experiment is considered as $T_{0}=0$ day. Then the equation consisting of the regression vector and regression matrix for measurements acquired from one mouse is

$$
\begin{aligned}
\left(\begin{array}{c}
\ln x\left(T_{0}\right) \\
\ln x\left(T_{1}\right) \\
\vdots \\
\ln x\left(T_{9}\right)
\end{array}\right)= & \left(\begin{array}{ccc}
1 & T_{0} & -10 / c\left(e^{-c T_{0}}-1\right) \\
1 & T_{1} & -10 / c\left(e^{-c T_{1}}-1\right) \\
\vdots & \vdots & \vdots \\
1 & T_{9} & -10 / c\left(e^{-c T_{9}}-1\right)
\end{array}\right) . \\
& \cdot\left(\begin{array}{c}
\ln x(0) \\
a \\
b
\end{array}\right) .
\end{aligned}
$$

If we denote the vector on the left-hand side of (17) made up from the measurements from mouse $i$ by $Y_{i}$ with $i=1,2,3,4$ (since there are measurements from four mice in the control group), and the matrix on the right-hand side of (17) by $X$, then the least squares estimation problem for measurements acquired from experiments from four mice can be written as

$$
\underbrace{\left(\begin{array}{l}
Y_{1} \\
Y_{2} \\
Y_{3} \\
Y_{4}
\end{array}\right)}_{Y}=\underbrace{\left(\begin{array}{c}
X \\
X \\
X \\
X
\end{array}\right)}_{\hat{X}} \underbrace{\left(\begin{array}{c}
\ln x(0) \\
a \\
b
\end{array}\right)}_{\beta}
$$

and the solution of the least squares estimation problem is

$$
\beta=\left(\hat{X}^{\top} \hat{X}\right)^{-1} \hat{X}^{\top} Y
$$

\section{RESULTS AND DISCUSSION}

The parametric identification carried out using the measurements on four mice from the control group resulted in the following parameters:

- $x(0)=45.46 \mathrm{~mm}^{3}$;

- $a=0.271 /$ day;

- $b=0.0074 \mathrm{~kg} /(\mathrm{mg} \cdot \mathrm{day})$.

The result of the simulation with the identified parameters and the same scenario as in the experiments in the control group is depicted in Fig. 1 along with the measurements from the four mice from the control group. The simulation result is in the convex hull of the measurements. Since the parameters resulted from least squares estimation based on (14), these result approximate the measurements best in least squares sense. 


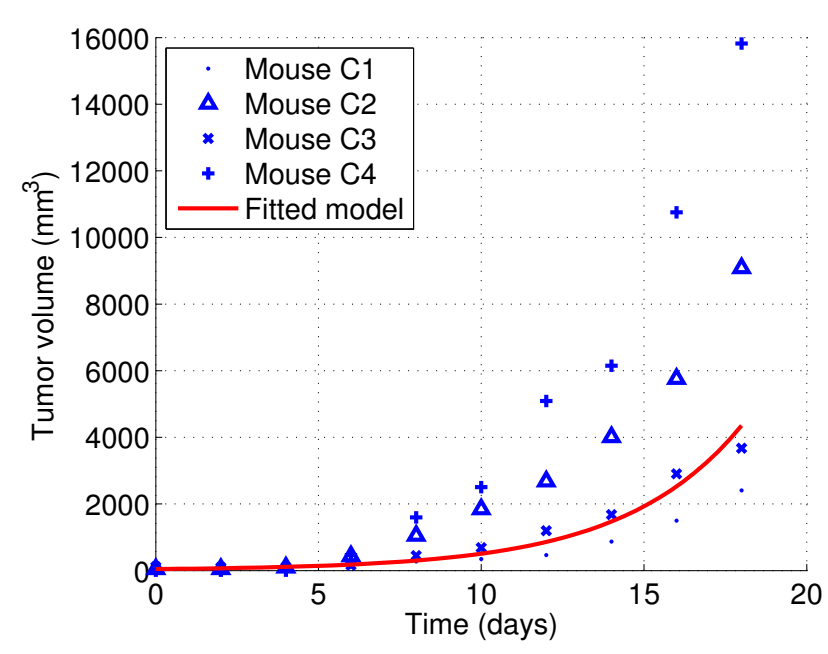

Fig. 1. The tumor volume measurements from the four mice from the control group, and the simulation result carried out with the minimal model with model parameters acquired with least squares estimation

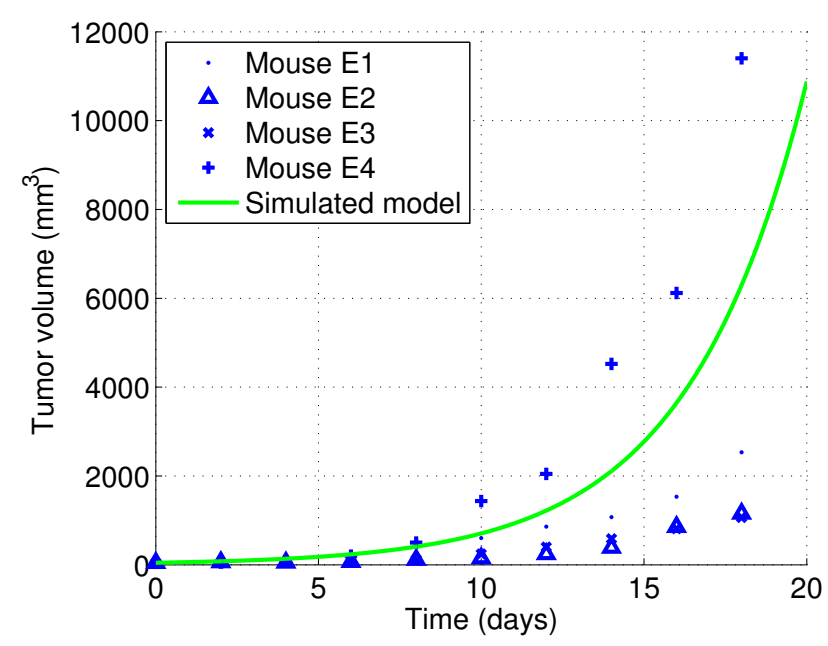

Fig. 2. The tumor volume measurements from four mice from the case group, and the simulation result carried out with the minimal model with model parameters acquired with least squares estimation

The same parameters were used to simulate the scenario for the case group, i.e. in which case $1 / 18 \mathrm{mg} / \mathrm{kg}$ bevacizumab was injected into the mice every day. The result of the simulation with measurements from four mice from the case group are in Fig. 2. The simulation result is in the convex hull of the measurements, showing that the model gives relevant results.

The results in Figs. 1 and 2 show that the proposed model can capture the dynamics of tumor growth under inhibition by bevacizumab. The performance of the model is good in the same scenario as the control group, since the measurements from the control group were used for parametric identification. However, the measurements from the case group were not taken into account in the identification process, the performance of the model is still good in the same scenario as the case group.

In our earlier works, linear models were identified for the growth of C38 colon adenocarcinoma in mice without treatment, and we have found that the tumor growth rates were $0.231 /$ day and $0.421 /$ day [11], however the $a=$ 0.231 /day growth rate was dominant. For the proposed model we acquired $a=0.271 /$ day with treatment here that is very close to the growth rate we acquired for the (different) measurements without treatment in [11]. In [12] identification of a linear tumor growth model was done for experiments with and without bevacizumab treatment. The tumor growth rate without treatment was found to be $a=0.291 /$ day, but it was $a=0.111 /$ day with treatment, since the effect of the inhibitor was taken into consideration by a linear term, so it was added to the rate of tumor growth. The application of the model proposed here solves this problem by using the bilinear term in (1), thus the effect of tumor growth and inhibition is clearly separated that can be seen in the values of the identified parameters as well, since the tumor growth rate with therapy is similar to the case without therapy.

\section{ACKNOWLEDGMENT}

This project has received funding from the European Research Council (ERC) under the European Union's Horizon 2020 research and innovation programme (grant agreement No 679681).

\section{REFERENCES}

[1] J. H. Distler, A. Hirth, M. Kurowska-Stolarska, R. E. Gay, S. Gay, and O. Distler, "Angiogenic and angiostatic factors in the molecular control of angiogenesis," The Quarterly Journal of Nuclear Medicine, vol. 47, no. 3, pp. 149-161, 2003.

[2] A. L. Harris, "Angiogenesis as a new target for cancer control," European Journal of Cancer Supplements, vol. 1, pp. 1-12, 2003.

[3] O. Distler, M. Neidhart, R. E. Gay, and S. Gay, "The molecular control of angiogenesis," International Reviews of Immunology, vol. 21, no. 1, pp. 33-49, 2002.

[4] N. Nath, T. Burg, D. M. Dawson, and E. Iyasere, "Optimizing antiangiogenic therapy for tumor minimization," in American Control Conference (ACC), 2010, June 2010, pp. 1242-1247.

[5] U. Ledzewicz and H. Schättler, "Analysis of optimal controls for a mathematical model of tumour anti-angiogenesis," Optimal Control Applications and Methods, vol. 29, no. 1, pp. 41-57, 2008. [Online]. Available: http://dx.doi.org/10.1002/oca.814

[6] J. Sápi, D. A. Drexler, I. Harmati, Z. Sápi, and L. Kovács, "Qualitative analysis of tumor growth model under antiangiogenic therapy: choosing the operating point and design parameters for controller design," Optimal Control Applications \& Methods, p. 19, 2015.

[7] L. Kovács, A. Szeles, J. Sápi, D. A. Drexler, I. Rudas, I. Harmati, and Z. Sápi, "Model-based angiogenic inhibition of tumor growth using modern robust control method," Computer Methods and Programs in Biomedicine, vol. 114, pp. e98-e110, 2014.

[8] J. Sápi, D. A. Drexler, and L. Kovács, "Comparison of mathematical tumor growth models," in In Proceedings of the IEEE 13th International Symposium on Intelligent Systems and Informatics, 2015, pp. 323-328.

[9] P. Hahnfeldt, D. Panigrahy, J. Folkman, and L. Hlatky, "Tumor development under angiogenic signaling: A dynamical theory of tumor growth, treatment response, and postvascular dormancy," Cancer Research, vol. 59, pp. 4770-4775, 1999.

[10] J.-B. Tylcz, K. E. Alaoui-Lasmaili, E.-H. Djermoune, N. Thomas, B. Faivre, and T. Bastogne, "Data-driven modeling and characterization of anti-angiogenic molecule effects on tumoral vascular density," Biomedical Signal Processing and Control, vol. 20, pp. 52-60, 2015. 
[11] J. Sápi, D. A. Drexler, I. Harmati, A. Szeles, B. Kiss, Z. Sápi, and L. Kovács, "Tumor growth model identification and analysis in case of c38 colon adenocarcinoma and b16 melanoma," in In Proceedings of the 8th IEEE International Symposium on Applied Computational Intelligence and Informatics, 2013, pp. 303-308f.

[12] J. Sápi, D. A. Drexler, Z. Sápi, and L. Kovács, "Identification of c38 colon adenocarcinoma growth under bevacizumab therapy and without therapy," in In Proceedings of the 15th IEEE International Symposium on Computational Intelligence and Informatics, 2014, pp. 443-448.

[13] J. Sápi, L. Kovács, D. A. Drexler, P. Kocsis, D. Gajári, and Z. Sápi, "Tumor volume estimation and quasi-continuous administration for most effective bevacizumab therapy," PLOS ONE, vol. 10, no. 11, pp. 1-20, 112015.

[14] P. Érdi and J. Tóth, Mathematical Models of Chemical Reactions. Theory and Applications of Deterministic and Stochastic Models. Princeton, New Jersey: Princeton University Press, 1989.

[15] D. A. Drexler and J. Tóth, "Global controllability of chemical reactions," Journal of Mathematical Chemistry, vol. 54, pp. 1327-1350, 2016.

[16] J. Sápi, T. Ferenci, D. A. Drexler, and L. Kovács, "Tumor model identification and statistical analysis," in In Proceedings of the 2015 IEEE International Conference on Systems, Man, and Cybernetics, 2015, pp. 2481-2486.

[17] F. Wu, M. Tamhane, and M. Morris, "Pharmacokinetics, lymph node uptake, and mechanistic pk model of near-infrared dye-labeled bevacizumab after iv and sc administration in mice," The AAPS Journal, vol. 14, no. 2, 2012. 
D. A. Drexler et al. • A minimal Model of Tumor Growth with Angiogenic Inhibition using Bevacizumab 